\title{
A DIFFERENTIALLY ALGEBRAIC REPLACEMENT THEOREM, AND ANALOG COMPUTABILITY
}

\author{
LEONARD LIPSHITZ AND LEE A. RUBEL
}

\begin{abstract}
A theorem is proved that enables one to replace a $C^{n}$ solution of a system of algebraic differential equations by analytic solutions nearby, such that each satisfies its own algebraic differential equation. As an application, we emend a proof of the Shannon-Pour-El thesis relating the outputs of analog computers to solutions of algebraic differential equations.
\end{abstract}

We prove here a differentially algebraic replacement theorem (DART for short) that says, roughly, that if a finite system $\Sigma$ of algebraic differential equations (ADE's for short) in the dependent variables $y_{1}, \ldots, y_{n}$ has a solution $\bar{y}_{1}(x), \ldots, \bar{y}_{n}(x)$ in a neighborhood of $x=a$, with the highest derivatives of the $\bar{y}_{j}$ demanded by $\Sigma$ being continuous, then $\Sigma$ has a solution $\overline{\bar{y}}_{1}(x), \ldots, \overline{\bar{y}}_{n}(x)$, where the $\overline{\bar{y}}_{j}$ are actually convergent power series in a neighborhood of $x=\bar{a}$ for suitable $\bar{a}$ arbitrarily close to $a$, and where the initial conditions on the $\overline{\bar{y}}_{j}$ at $\bar{a}$ are exactly the initial conditions on the $\bar{y}_{j}$ at $\bar{a}$. Moreover, the $\overline{\bar{y}}_{j}$ may all be chosen to be differentially algebraic (DA for short), which means that each $\overline{\bar{y}}_{j}$ satisfies an ADE in which only that $y_{j}$ (and no other $y_{i}$ ) occurs. The proof of DART is via elimination theory.

As an application of DART, we give a proof of Theorem 2 of [POE]. The proof offered in [POE] has some gaps in it, which we describe later in this paper before giving our own proof. Because Theorems 2 and 4 of [POE] together establish a fundamental connection between analog computers and algebraic differential equations, we consider providing a complete proof to be important. (See [SHA] for an earlier, but incomplete, version of this connection.) Professor Pour-El has communicated to us that she has repairs for the proof in [POE].

THEOREM 1 (DART). Let $\bar{y}_{i} \in C^{n_{i}}, i=1, \ldots, m$, be functions on an interval $L \subseteq \mathbf{R}$. Let $I$ be the set of all differential polynomials $f$, with coefficients from $\mathbf{R}[x]$, in the variables $y_{i}$ such that the order of $f$ in $y_{i}$ is $\leqslant n_{i}$ for $i=1, \ldots, m$ and such that $f(\bar{y})=0$ for all $x \in L$ where $f(\bar{y})$ means

$$
f(\bar{y}(x))=f\left(x, \bar{y}_{1}(x), \bar{y}_{1}^{\prime}(x), \ldots, \bar{y}_{1}^{\left(n_{1}\right)}(x), \ldots, \bar{y}_{m}(x), \ldots, \bar{y}_{m}^{\left(n_{m}\right)}(x)\right) .
$$

Received by the editors October 3, 1984 and, in revised form, January 3, 1986.

1980 Mathematics Subject Classification (1985 Revision). Primary 03 B60.

The authors' research was partially supported by separate grants from the National Science Foundation. The authors would like to thank Peter Lindsay, Bernard Johnston, and the referee for suggestions which improved this paper. 
Let $\pi$ be a finite set of differential polynomials of order $\leqslant n_{i}$ in $y_{i}$ for $i=1, \ldots, m$ such that if $f \in \pi$ then $f(\bar{y})$ does not vanish identically on any nontrivial subinterval of $L$. Then there exists an $a \in L$ and differentially algebraic functions $\tilde{y}_{i}, i=1, \ldots, m$, analytic at $x=a$ such that $\tilde{y}_{i}^{(j)}(a)=\bar{y}_{i}^{(j)}(a)$ for $i=1, \ldots, m$ and $j=0, \ldots, n_{i}$ and such that $f(\tilde{y})=0$ for every $f \in I$ for all $x$ near $a$ and $f(\tilde{y})(a) \neq 0$ for all $f \in \pi$. In fact, the set of such $a$ 's is dense in $L$.

Proof. Let the $y_{i}^{(j)}$ be indeterminates and consider the polynomial rings

$$
\begin{aligned}
R & =\mathbf{R}\left[x,\left\{y_{i}^{(j)}: 1 \leqslant i \leqslant m, 0 \leqslant j \leqslant n_{i}\right\}\right], \\
R^{\prime} & =\mathbf{R}\left[x,\left\{y_{i}^{(j)}: 1 \leqslant i \leqslant m, 0 \leqslant j<n_{i}\right\}\right],
\end{aligned}
$$

so that

$$
I=\{f \in R: f(\bar{y}) \equiv 0 \text { on } L\} .
$$

(When $f \in R$ and $\bar{y}=\left(\bar{y}_{1}(x), \ldots, \bar{y}_{n}(x)\right)$ are functions on $L$ with $\bar{y}_{i} \in C^{n_{i}}$ for each $i$, then by $f(\bar{y})$ we mean the function on $L$ obtained by substituting the $\bar{y}_{i}^{(j)}$ for the variables $y_{i}^{(j)}$ in $f$.) We must produce an $a \in L$ and $\tilde{y}_{1}, \ldots, \tilde{y}_{m}$ such that

(0) each $\tilde{y}_{i}$ is analytic and differentially algebraic at $a$,

(1) $\tilde{y}_{i}^{(j)}(a)=\bar{y}_{i}^{(j)}(a)$ for each $1 \leqslant i \leqslant m, 0 \leqslant j \leqslant n_{i}$,

(2) $(\forall f \in I)[f(\tilde{y})=0$ for $x$ near $a]$, and

(3) $(\forall f \in \pi)[f(\tilde{y})(a) \neq 0]$.

First of all, we claim that we may assume without loss of generality that $I$ is a prime ideal in $R$ and that $(\forall f \notin I)[f(\bar{y})$ does not vanish identically on any nontrivial subinterval of $L$ ]. For suppose there exists $f_{1} \in R \backslash I$ such that $f_{1}(\bar{y})$ vanishes identically on an open, nontrivial subinterval $L_{1}$ of $L$. Let $I_{1}$ be the ideal $\left\{f \in R: f(\bar{y}) \equiv 0\right.$ on $\left.L_{1}\right\}$. Then $I \subsetneq I_{1} \varsubsetneqq R$. The first containment is proper because $f_{1} \in L_{1}$ and the second because $1 \notin I_{1}$. Iterate this construction. Because $R$ is noetherian, the process stops after a finite number of steps; at $L_{k}$ and $I_{k}$, say. Thus $\left(\forall f \in R \backslash I_{k}\right)[f(\bar{y})$ does not vanish identically on any nontrivial subinterval of $L_{k}$ ]. We can also see that $I_{k}$ is a prime ideal. For if $f g \in I_{k}$ but $f \notin I_{k}$, then $f(\bar{y})$ does not vanish on any nontrivial subinterval of $L_{k}$ so by continuity $g(\bar{y})$ must vanish identically on $L_{k}$ and $g \in I_{k}$. This proves the claim.

For $m$-tuples $\vec{n}=\left(n_{1}, \ldots, n_{m}\right)$ let $|\vec{n}|=n_{1}+\cdots+n_{m}$. We shall prove the theorem by induction on $|\vec{n}|$.

Case A: $I \cap R^{\prime}=(0)$. (We remark as an aside that this is the case for the beginning of the induction $\vec{n}=(0, \ldots, 0)$, and that this part of the proof does not use the induction hypothesis.) In this case, we think of $R^{\prime}$ as a subring of $R / I$. Reorder the indices $i$ so that, in $R / I,\left\{y_{i}^{\left(n_{i}\right)}\right\}_{1 \leqslant i \leqslant r}$ are algebraically independent over $R^{\prime}$ and each $y_{i}^{\left(n_{i}\right)}$ with $i>r$ is algebraic over $R^{\prime}\left[\left\{y_{i}^{\left(n_{i}\right)}: 1 \leqslant i \leqslant r\right\}\right]$ in $R / I$, using $y_{i}^{(j)}$ to also denote the image of $y_{i}^{(j)}$ in $R / I$. Let $P_{k}$ be a polynomial of minimal degree such that $y_{k}^{\left(n_{k}\right)}$ satisfies $P_{k}(y)=0$ in $R / I$ and such that the coefficients of $P_{k}$ are in $R^{\prime}\left[\left\{y_{j}^{\left(n_{j}\right)}: 1 \leqslant j<k\right\}\right]$.

Let $S_{k}=\partial P_{k} / \partial y_{k}^{\left(n_{k}\right)}$ (the "separant" of $P_{k}$ ), let $d_{k}=$ degree of $P_{k}$ in $y_{k}^{\left(n_{k}\right)}$, and let $C_{k}$ be the leading coefficient of $P_{k}$. From the definition of $P_{k}$ we see that $S_{k} \notin I$ and $C_{k} \notin I$. Let $G=\prod_{k=r+1}^{m} C_{k} S_{k}$. Then $G \notin I$ since $I$ is prime. 
We claim that for any $\tilde{y}=\left(\tilde{y}_{1}, \ldots, \tilde{y}_{m}\right)$ with the $\tilde{y}_{i} \in C^{n_{i}}$, and any interval $X$

$$
\begin{aligned}
& \text { if } P_{r+1}(\tilde{y})(x)=\cdots=P_{m}(\tilde{y})(x)=0 \text { and } G(\tilde{y})(x) \neq 0 \forall x \in X, \\
& \text { then } f(\tilde{y})(x)=0 \forall f \in I \text { and } \forall x \in X .
\end{aligned}
$$

To prove this, let $f \in I$ and note that by the Euclidean algorithm, there is an $H \in R$ which is a product of $C_{i}$ 's and $S_{j}$ 's such that

$$
H f=h_{r+1} P_{r+1}+\cdots+h_{m} P_{m}+Q
$$

where the degree of $y_{k}^{\left(n_{k}\right)}$ in $Q$ is less than $d_{k}$ for each $k$ with $r<k \leqslant m$. Consider $k=m$. The left-hand side of $(*)$ vanishes in $R / I$ and the right-hand side is a polynomial in $y_{m}^{\left(n_{m}\right)}$. Therefore by definition of $P_{m}$, the degree of $y_{m}^{\left(n_{m}\right)}$ in $Q$ must be zero. Repeating this argument $m$ times shows that $Q \equiv 0$. This proves the above claim.

By the choice of $\pi$ and our first claim we see that for all $f \in \pi^{\prime}=\pi \cup\{G\}$ we have that $f(x, \bar{y}(x))$ vanishes identically on no nontrivial subinterval of $L$. Let $\mathscr{S}=\left\{a \in L: f(x, \bar{y}(x))(a) \neq 0\right.$ for all $\left.f \in \pi^{\prime}\right\}$. Then $\mathscr{S}$ is dense in L. Let $a \in \mathscr{S}$, and let

$$
\tilde{y}_{i}= \begin{cases}\sum_{j=0}^{n_{i}}\left(\frac{\bar{y}_{i}^{(j)}(a)}{j !}\right)(x-a)^{j} & \text { for } 1 \leqslant i \leqslant r, \\ \sum_{j=0}^{n_{i}}\left(\frac{\bar{y}_{i}^{(j)}(a)}{j !}\right)(x-a)^{j}+\sum_{j>n_{i}} a_{i j}(x-a)^{j} & \text { for } r<i \leqslant m,\end{cases}
$$

where the $a_{i j}$ 's are determined by the following triangular system of $m-r$ differential equations in the unknowns $y_{r+1}, \ldots, y_{m}$ :

$$
\left\{\begin{array}{l}
P_{r+1}\left(\tilde{y}_{1}, \ldots, \tilde{y}_{r}, y_{r+1}\right)=0 \\
\vdots \\
P_{m}\left(\tilde{y}_{1}, \ldots, \tilde{y}_{r}, y_{r+1}, \ldots, y_{m}\right)=0
\end{array}\right.
$$

together with the initial condition $y_{j}^{(k)}(a)=\bar{y}_{j}^{(k)}(a)$ for $j=r+1, \ldots, m ; k=$ $0, \ldots, n_{j}$. (This system has the Jacobian separant

$$
\left|\left(\frac{\partial P_{r+i}}{\partial h_{r+j}}\right)(a)\right|=\left|\begin{array}{ccc}
S_{r+1}(\bar{y})(a) & & 0 \\
? & \ddots & \\
& & S_{m}(\bar{y})(a)
\end{array}\right|=S(\bar{y}(a)) \neq 0,
$$

where $h_{k}=y_{k}^{\left(n_{k}\right)}$. Hence, by the Fundamental Existence Theorem, this system plus initial conditions has a unique, analytic, solution $\tilde{y}$ near $a$.)

We claim that these $\tilde{y}_{i}$ 's have the required properties.

(0) $\tilde{y}_{1}, \ldots, \tilde{y}_{r}$ are polynomials, so they certainly satisfy (0). The others satisfy a system of ADE's, with separant Jacobian $\neq 0$, so by [RUB], they are each differentially algebraic. (One can also see this by noting that the transcendence degree of $\mathbf{R}\left(x, \tilde{y}_{r}, \tilde{y}_{r}^{\prime}, \ldots, \tilde{y}_{m}, \tilde{y}_{m}^{\prime} \cdots\right)$ over $\mathbf{R}(x)$ is finite and hence all the $\tilde{y}_{i}$ are differentially algebraic over $\mathbf{R}(x)$.)

(1) holds by construction,

(2) follows from (\#),

(3) holds since $f(\tilde{y})(a)=f(\bar{y}(a)) \neq 0$ for all $f \in \pi$. 
This completes Case A.

Case B: $I \cap R^{\prime} \neq(0)$. Let $\vec{n}^{\prime \prime}=\left(n_{1}^{\prime \prime}, \ldots, n_{m}^{\prime \prime}\right)$ be an integer vector with $0 \leqslant n_{i}^{\prime \prime}<$ $n_{i}$ for $i=1, \ldots, m$, such that $R_{\vec{n}^{\prime \prime}} \cap I \neq(0)$, where

$$
R_{\vec{n}^{\prime \prime}}=\mathbf{R}\left[x,\left\{y_{i}^{(j)}: 1 \leqslant i \leqslant m, 0 \leqslant j \leqslant n_{i}^{\prime \prime}\right\}\right] .
$$

Take $0 \neq f \in I \cap R_{\vec{n}^{\prime \prime}}$ of lowest total degree. Then $f$ is irreducible since $I$ is prime, and $f \in R^{\prime}$. Let

$$
k_{i}= \begin{cases}\text { order of } f \text { in } \dot{y}_{i}, & \text { if } y_{i} \text { appears in } f \\ -1 & \text { otherwise }\end{cases}
$$

Let

$$
\delta=\min \left\{n_{i}-k_{i}: k_{i} \neq-1,1 \leqslant i \leqslant m\right\},
$$

which is achieved at $i_{0}$, say. Then $\delta \geqslant 1$. Let $S=\partial f / \partial y_{i_{0}}^{\left(k_{i_{0}}\right)}$. Then $S \notin I$ (by the definition of $f$ ). From our very first claim, $S(\bar{y})$ does not vanish identically on any nontrivial subinterval of $L$. Differentiating the equation " $f=0$ " $\delta$ times with respect to $x$ gives

$$
S y_{i_{0}}^{\left(n_{i_{0}}\right)}+T=0
$$

where $T \in R_{\vec{n}}$, and $\vec{n}^{\prime}=\left(n_{1}, \ldots, n_{i_{0}}-1, \ldots, n_{m}\right)$. (Notice that $d f / d x=\partial f / \partial x+$ $\sum\left(\partial f / \partial y_{i}^{(j)}\right) y_{i}^{(j+1)}$.) The idea now is to formally substitute $y_{i_{0}}^{\left(n_{i_{0}}\right)}=-T / S$ in our ADE's and multiply by suitable powers of $S$ to clear the denominators. More precisely, let $A=\left\{h \in R_{\vec{n}^{\prime}}: h\right.$ is obtained from $S^{\alpha} g$, where $\alpha \geqslant 0$ and $g \in I \backslash R_{\vec{n}^{\prime}}$, by using $(\dagger)$ to get rid of the $y_{i_{0}}^{\left(n_{i_{0}}\right)}$ terms $\}$. Construct $\pi^{\prime}$ from $\pi \cup\{S\}$ in a similar way, and let $I^{\prime}$ be the ideal in $R_{\vec{n}^{\prime}}$ generated by $A \cup\left(R_{\vec{n}^{\prime}} \cap I\right)$. Note that $f \in I^{\prime}$. Now apply the induction hypothesis to $I^{\prime}$ and $\pi^{\prime}$ in $R_{\vec{n}^{\prime}}$ to get $a \in L, \tilde{y}_{1}, \ldots, \tilde{y}_{m}$ satisfying (0)-(3) above for $I^{\prime}, \pi^{\prime}$. We claim that $a$ and these $\tilde{y}_{i}$ also satisfy (0)-(3) for $I$ and $\pi$.

$(0)$ is automatic.

For (1), we need only prove the case $i=i_{0}$ and $j=n_{i_{0}}$, the others being automatic. Now $S(\tilde{y}) \tilde{y}_{i_{0}}^{\left(n_{i_{0}}\right)}+T(\tilde{y}) \equiv 0$ near $a$ and $S(a, \tilde{y}(a)) \neq 0$. Thus

$$
\tilde{y}_{i_{0}}^{\left(n_{i_{0}}\right)}(a)=(-T / S)(\tilde{y}(a))=(-T / S)(\bar{y}(a))=\bar{y}_{i_{0}}^{\left(n_{i_{0}}\right)}(a) .
$$

(The second equation follows from (1).) (2) and (3) are proved similarly. Replacing $L$ by arbitrary subintervals of $L$ we see that the set of $a$ 's with the required property is dense in $L$ and the proof of DART is complete.

We now discuss Theorem 2 of [POE].

THEOREM 2 (POUR-EL). If $y$ is generable on an interval I by an analog computer, then there is an interval $I^{\prime} \subseteq I$ such that on $I^{\prime}, y$ satisfies an algebraic differential equation. 
Pour-El proceeds as follows (we quote, in part). There are functions $y_{2}, \ldots, y_{n}$ which satisfy a set of equations of the form

$$
A(x, \vec{y}) \frac{d \vec{y}}{d x}=\vec{B}(x, \vec{y}),
$$

where $y=y_{2}, \vec{y}$ is the column vector with components $y_{2}, \ldots, y_{n} ; A$ is an $(n-1) \times$ $(n-1)$ matrix whose components are linear in $1, x, y_{2}, \ldots, y_{n}$, and $\vec{B}$ is an $(n-1)$-dimensional vector whose components are linear in $1, x, y_{2}, \ldots, y_{n}$. Further, the system (E) is supposed to be provided with initial conditions $y_{2}(a)=$ $y_{2}^{*}, \ldots, y_{n}(a)=y_{n}^{*}$ so that (E) has a unique solution locally, even when $a$ and $y_{2}^{*}, \ldots, y_{n}^{*}$ are slightly perturbed.

In the language of analog computers, the system (E) specifies the outputs of all the integrators, and the further assumptions about the initial conditions (called "domain of generation" in [POE]) say that the computer is deterministic even if the operator makes small errors in the initial settings of the integrators. This illuminating condition of Pour-El's seems to be central to the connection between analog computers and algebraic differential equations.

The error seems to be made in Case 1 in the proof of Theorem 2 in [POE] in considering $A$ as an abstract matrix and not, as it must be, constrained to follow along the given solution $y(x)$. Later, in Case 2, the domain-of-generation hypothesis about the original system is apparently incorrectly applied to an altered system. In view of this, it seems desirable to provide our alternative proof by way of DART.

ProOF OF THEOREM 2 OF [POE]. We can actually get by with a weaker hypothesis than the full domain-of-generation assumption, namely that there exist an open interval $N$ around $x=a$ such that for all $\bar{a} \in N$, the equations (E) with the initial conditions

$$
\begin{aligned}
& y_{2}(\bar{a})=\bar{y}_{2}(\bar{a}), \ldots, y_{2}^{\left(n_{2}\right)}(\bar{a})=\bar{y}_{2}^{\left(n_{2}\right)}(\bar{a}), \\
& \quad \vdots \\
& y_{m}(\bar{a})=\bar{y}_{m}(\bar{a}), \ldots, y_{m}^{\left(n_{m}\right)}(\bar{a})=\bar{y}_{m}^{\left(n_{m}\right)}(\bar{a}),
\end{aligned}
$$

(where $\overrightarrow{\bar{y}}$ is the solution for the initial conditions at $\bar{a}$ ) have a locally unique solution, i.e., a solution in all small intervals around $\bar{a}$. In other words, we need allow small errors in the time setting only, and not in the setting of the integrators also. Now apply DART to the solution $\overrightarrow{\vec{y}}$ to get a differentially algebraic analytic solution $\overrightarrow{\tilde{y}}$ in a neighborhood of a nearby point $\bar{a}$ on $\mathbf{R}$, with $\overrightarrow{\tilde{y}}$ having the same initial conditions at $\bar{a}$ as $\overrightarrow{\vec{y}}$. Notice that the $\bar{y}_{i}$ satisfy the $C^{n_{j}}$ hypothesis of DART (here $n_{j}=1$ for all $j$ ) because, throughout [POE], only $C^{1}$ solutions of the system (E) are considered. By the above uniqueness assumptions, we must have $\bar{y}=\tilde{y}$ throughout a small neighborhood of $\bar{a}$. It follows that $y_{2}$ is differentially algebraic in that neighborhood, and the theorem is proved.

We have been unable to determine whether the still weaker uniqueness hypothesis will suffice, that just for the initial conditions $y_{2}(a)=y_{2}^{*}, \ldots, y_{m}(a)=y_{m}^{*}$, the system (E) has a (locally) unique solution. It is surely not correct that such a hypothesis implies domain of generation for just any system of ADE's, but it could conceivably be true for systems of the special form (E). 
Finally, we raise the question whether there is an algorithm for determining whether a given system (E), with given initial conditions, has a domain of generation. This is essentially equivalent to the question of finding a procedure for deciding, for a given analog computer, whether it will run for a positive length of time.

\section{REFERENCES}

[POE] Marian Boykan Pour-El, Abstract computability and its relation to the general purpose analog computer (Some connections between logic, differential equations and analog computers), Trans. Amer. Math. Soc. 199 (1974), 1-29.

[RUB] Lee A. Rubel, An elimination theorem for systems of algebraic differential equations, Houston J. Math. 8 (1982), 289-295.

[SHA] Claude E. Shannon, Mathematical theory of the differential analyzer, J. Math. Phys. Mass. Inst. Tech. 20 (1941), 337-354.

DePartment of Mathematics, Purdue University, West Lafayette, Indiana 47907

DEPARTMENT OF MATHEMATICS, UNIVERSITy OF ILLINOIS, URBANA, IllinOIS 61801 